\title{
ANALYSIS OF THE INFLUENCE OF MODES OF PLASMA- CHEMICAL SYNTHESIS AND PRECURSOR EVAPORATION ON GEOMETRIC CHARACTERISTICS OF CERAMIC POWDERS PRODUCED
}

\author{
Ivan S. Bondarchuk ${ }^{1}$, Ilya A. Zhukov ${ }^{1}$, Boris V. Borisov ${ }^{2 a}$ \\ ${ }^{1}$ National Research Tomsk State University, SRIAMM, 634050 Tomsk, Russia \\ ${ }^{2}$ National Research Tomsk Polytechnic University, 634050 Tomsk, Russia
}

\begin{abstract}
Mathematical model of evolution of a liquid precursor droplet in the process of plasma-chemical transformations for production of micro- and nano-structured ceramic powders on the basis of metal oxides is described. A criterion of production of particles with different morphology is determined. The results of numerical analysis are given.
\end{abstract}

\section{INTRODUCTION}

Surface reinforcement using micro- and nano-structured ceramic coatings is a very important area in modern industry. Metal oxide ceramic powders have proven to be a perfect material for this purpose. Development of technologies for their production is an important objective. Along with economic efficiency the production technology must make it possible to control end-product morphology. This concept includes stable production of powder in a specified (as narrow as possible) range of particle size having the form of solid or hollow spheres. These powder parameters in the process of application to surfaces will be able to provide a wide range of coating parameters: from a dense film to a thermal insulation layer with an extensive system of closed pores.

Plasma-chemical technology is considered in the framework of this research, the basis of this technology is the process of high-temperature decomposition of water solutions of salts obtaining ultrafine (nano-) powders of simple and complex oxides[1]. The process of plasma-chemical synthesis is a multi-stage one and includes spraying of a liquid precursor with generation of polydisperse drops in reactor chamber, movement of particles in the flux of gas heat transfer medium, heating and evaporation of drops, followed by diffusion and crystallization of salt in drop volume, salt thermolysis along with formation of a microporous metal oxide film under increasing temperature and its sintering in the form of a solid hollow particle.

Particularly when water solution of aluminum nitrate nonahydrate $\mathrm{Al}\left(\mathrm{NO}_{3}\right)_{3} \cdot 9 \mathrm{H}_{2} \mathrm{O}$ is used as a precursor in thermolysis processes while heating up to $135^{\circ} \mathrm{C}$ and higher and in the process of further decomposition of obtained product at the temperature higher than $200^{\circ} \mathrm{C}$ end-product $\mathrm{Al}_{2} \mathrm{O}_{3}$ is obtained according to the following reaction:

Mathematical modeling of thermal processes in the considered design avoids unnecessary costs in establishing the most effective modes of technology acquisition and development of high-silicate melt

${ }^{\mathrm{a} C}$ Corresponding author: bvborisov@tpu.ru 
from the waste of energy and mining industry using the energy of low-temperature plasma. In this case, the total cross-sectional area, free to move in this direction of the flow varies from a minimum at the entrance to the flow of raw materials up to a maximum at the intersection of the axis of the cylindrical crucible and again to a minimum at the expiration of the finished melt (Fig. 2).

In accordance with a change in the geometry of the flow cross section and the flow rate varies. The minimum radial velocity is in the axis of the crucible, where the stream is most intense thermal effect, which favorably affects the final result, in terms of the completeness of the greatest melt. On the other hand achieve a melt temperature higher than necessary, leading to thermal decomposition of the raw material required to loss of useful properties. Preliminary analysis of the modes of fusion by means of mathematical modeling, taking into account the maximum design features allowing for an early design stage to choose the most rational modes of loading of raw materials, melt and discharge parameters of the plasma column.

$$
4 \mathrm{Al}\left(\mathrm{NO}_{3}\right)_{3}=2 \mathrm{Al}_{2} \mathrm{O}_{3}+12 \mathrm{NO}_{2} \uparrow+3 \mathrm{O}_{2} \uparrow .
$$

Analysis of plasma-chemical synthesis indicated that powder particle morphology is formed at the stage of droplet evaporation and determined by simultaneous processes taking place in the droplet: heating and evaporation of solution, diffusion of salt to the center of the droplet, salt crystallization and removal of solvent residues (droplet drying). Full mathematical model of considered processes for analysis and selection of the most efficient parameters of plasma-chemical technology includes the tasks of calculation of heat transfer medium flow field and movement of liquid precursor particles. The flow of gas and condensed particles was considered stationary and axially symmetric; turbulent shear stresses were determined by $\mathrm{k}-\varepsilon$-model constraints, the impact of solution drops on turbulence structure was neglected; there were no heat transfer between the gas and channel borders; flow field was considered taking into account heat, mass and momentum exchange between droplets (particles) and gas-vapor flow [1].

\section{THEORETICAL}

Droplet evolution is considered assuming it has spherical form, constant thermophysical parameters and transfer characteristics of solution and solvent and close-to-zero impact of thermodiffusion during the whole process. Analysis of Biot numbers for heat and mass transfer processes indicated that the droplet under considered conditions has the property of thermally slender body; however, salt concentration distribution over the droplet volume cannot be neglected [2].

Averaged value of droplet temperature $T$ to saturation (boiling) temperature $T_{\text {vap }}$ is determined by the following equation:

$$
\frac{d T}{d t}=\frac{3}{2} \frac{a}{R^{2}} \mathrm{Nu}\left(T_{e}-T\right)
$$

where $a$ - solution thermometric conductivity coefficient, $T_{e}$ - flow temperature, $\mathrm{Nu}$ - Nusselt numbers, $R$ - droplet outer radius. Further thermal influence of the flow on the particle leads to droplet size reduction due to solvent evaporation which is simulated in the framework of reduced film model [3]:

$$
\frac{d R}{d t}=-\frac{\lambda_{g}}{2 \rho R c_{\text {vap }}} \mathrm{Nu} \cdot \ln (1+\mathrm{B}),
$$

where $B=c_{\text {vap }}\left(T_{e}-T_{\text {vap }}\right) / L$ - Spalding number; $\lambda_{g}$ - gas heat conductivity coefficient; $c_{\text {vap }}-$ specific heat capacity; $L$ - heat of vaporization .

The change of salt concentration in droplet solution is described by the following diffusion equation: 


$$
\frac{\partial C}{\partial t}=\frac{1}{r^{2}} \frac{\partial}{\partial r}\left(r^{2} D_{S} \frac{\partial C}{\partial r}\right)
$$

where $D_{S}$ - diffusion coefficient. Initial conditions for heat conductivity and diffusion equations: equal initial concentration and temperature for the whole droplet volume. Concentration field in the center of the droplet is considered symmetrical. Up to the moment when droplet temperature reaches the value $T=T_{\text {vap }}$ heat exchange on its surface is simulated by the third kind boundary condition. At the stage of intensive evaporation of solution the parameters of mass exchange process at moving boundary $r=R(t)$ are determined by the equality of temperatures $T=T_{\text {vap }}$ and mass conservation law for the salt at moving surface:

$$
D_{S} \frac{\partial C}{\partial r}=-\rho \frac{\partial R}{\partial t} .
$$

Analysis of equations (1) and (2) makes it possible to introduce a dimensionless parameter equal to the ratio of characteristic time of salt diffusion in droplet volume to characteristic (according to reduced film model) time of evaporation of liquid from its surface:

$$
K=\frac{\lambda_{g}}{2 \rho D_{S} c_{\text {vap }}} \mathrm{Nu} \cdot \ln (1+\mathrm{B}) .
$$

\section{Results and discussion}

A droplet low-concentration water solution of metal salt with the initial radius $\mathrm{R} 0=25 \mu \mathrm{m}$ was considered as an example. At atmospheric pressure and boiling temperature the following values of basic parameters were determined:

$$
\begin{array}{llc}
\mathrm{D}_{\mathrm{s}}=10^{-9} \mathrm{~m}^{2} / \mathrm{s} & =10^{3} \mathrm{~kg} / \mathrm{m}^{3}, & a=1, .58 \cdot 10^{-9} \mathrm{~m}^{2} / \mathrm{s}, \\
C_{\text {vap }}=2,0 \cdot 10^{3} & L=2,26 \cdot 10^{6} \mathrm{~J} / \mathrm{kg}, & \mathrm{Nu}=2 \\
\mathrm{~J} /(\mathrm{kg} \cdot \mathrm{K}), & T_{\text {vap }}=373 \mathrm{~K}, & g=0,6 \mathrm{~kg} / \mathrm{m}^{3}, \\
T_{e}-T_{\text {vap }}=50 \mathrm{~K}, & g=2,2 \cdot 10^{-5} \mathrm{~Pa} \cdot \mathrm{s} . & \\
\square g=3,3 \cdot 10^{-2} & & \\
\mathrm{~W} /(\mathrm{m} \cdot \mathrm{K}), &
\end{array}
$$

The results of calculation of fields of dimensionless concentrations $x$ for different values of dimensionless times $\tau$ and values of criterion $\mathrm{K}$ are shown in Fig.1:

$$
\tau=t \frac{D_{S}}{R_{0}^{2}}, x=\frac{C}{C_{k r}},
$$

where $C_{k r}$ - experimental critical concentration value, if this value is exceeded salt encrustation begins.
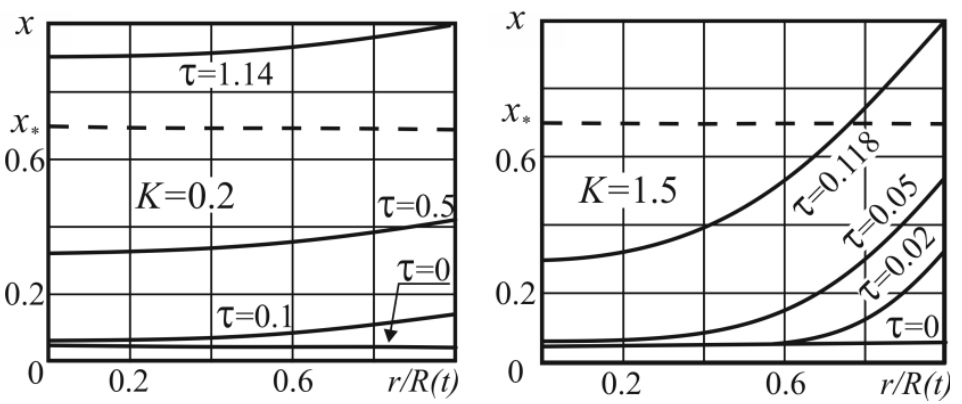

Figure 1. Distribution of salt concentration in the droplet as a function of time 
As the analysis of calculations and experimental data indicates, formation of "nut"-type particles with an outer surface shell and inner cavity takes place at $K>0.75$. An example of such experimentally obtained "nut" according to TEM data is shown in Fig.2.

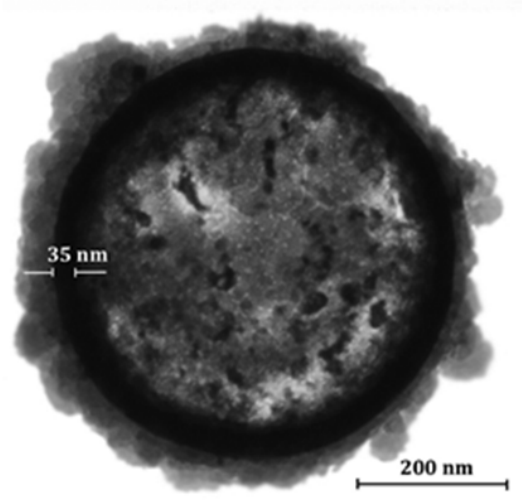

Figure 2. Example of structure and sizes of powder produced

\section{Conclusion}

Temperature difference between heat transfer medium and droplet imposes a major impact on $\mathrm{K}$ parameter at fixed values of thermophysical characteristics of substances (salt and solvent). If the temperature difference is small - solid particles are to be formed, otherwise - hollow particles are to be formed. When the temperature difference is significant evaporation process is quicker than diffusion (K parameter increases), crystallization takes place in subsurface layer only. And vice versa when the temperature difference is small salt concentration reaches equilibrium value throughout the whole droplet volume..

This work was supported by the Ministry of Education and Science of the Russian Federation under the Federal Target Program "Research and development on priority directions of scientifictechnological complex of Russia for 2014-2020", the agreement № 14.578.21.0034, unique identifier PNI RFMEFI57814X0034 and grant № 8.2.46.2015 "The Tomsk State University Academic D.I. Mendeleev Fund Program"

\section{References}

1. A.S. Zhukov, V.A. Arkhipov, S.S. Bondarchuk. Heat and mass transfer in the process of production of metal oxides by means of plasma-chemical method// Izvestiya vuzov. Physics. 2010. Vol. 53. № 12 (2). P. 121-126. (in Russian)

2. I.S. Bondarchuk, A.S. Zhukov. Criterion of particle shape formation in the process of plasmachemical synthesis of powders. // Chemical physics and contemporary issues of power engineering / Collection of papers of All-Russian youth conference. National Research Tomsk Polytechnic University. Institute of Power Engineering. Tomsk, 2012. P. 34-38.(in Russian)

3. Practical combustion theory basics: Study Guide for universities/ V.V.Pomerantsev, K.M. Arefyev, D.B. Akhmedov et. all; Editor: V.V. Pomerantsev. -Leningrad.:Energoatomizdat, 1986. -315 p. (in Russian) 